\title{
BMJ Open Addressing HIV care, mental health and substance use among youth and young adults in the Bay Area: description of an intervention to improve information, motivation and behavioural skills
}

Caravella McCuistian (1) , ${ }^{1}$ Angie R Wootton (1) , ${ }^{2}$ Dominique Legnitto-Packard, ${ }^{3}$ Valerie A. Gruber, ${ }^{1}$ Carol Dawson-Rose, ${ }^{4}$ Mallory O. Johnson, ${ }^{3}$ Parya Saberi (i) ${ }^{3}$

To cite: McCuistian C, Wootton AR, LegnittoPackard D, et al. Addressing HIV care, mental health and substance use among youth and young adults in the Bay Area: description of an intervention to improve information, motivation and behavioural skills. BMJ Open 2021;11:e042713. doi:10.1136/ bmjopen-2020-042713

- Prepublication history and additional material for this paper are available online. To view these files, please visit the journal online (http://dx.doi org/10.1136/bmjopen-2020042713).

Received 14 July 2020 Revised 09 February 2021 Accepted 09 March 2021

Check for updates

(C) Author(s) (or their employer(s)) 2021. Re-use permitted under CC BY-NC. No commercial re-use. See rights and permissions. Published by BMJ.

For numbered affiliations see end of article.

Correspondence to Dr Caravella McCuistian; Caravella.Mccuistian@ucsf.edu

\section{ABSTRACT}

Objective Youth represent a population disparately impacted by the HIV epidemic. With most new HIV diagnoses occurring among adolescents and young adults, novel approaches to address this disparity are necessary. The objective of the current study was to describe the Youth to Telehealth and Text to Improve Engagement in Care (Y2TEC) intervention, which aims to fill this gap. The Y2TEC intervention (trial registration NCT03681145) offers an innovative approach to improve HIV treatment engagement among youth living with HIV by focusing on treatment barriers related to mental health and substance use. This allows for a holistic approach to providing culturally informed intervention strategies for this population.

Participants and setting The Y2TEC intervention was developed for youth with HIV in the large metropolitan area of the San Francisco Bay Area. The Y2TEC intervention was developed based on formative interdisciplinary research and is grounded in the information-motivationbehavioural skills model.

Results The intervention includes 12 sessions each lasting 20-30 minutes, which are delivered through videoconferencing and accompanying bidirectional text messaging. The intervention sessions are individualised, with session dosage in each major content area determined by participant's level of acuity.

Conclusions The Y2TEC intervention is well positioned to help decrease HIV-related disparities in youth living with HIV through its innovative use of video-counselling technologies and an integrated focus on HIV, mental health and substance use.

\section{INTRODUCTION}

While evidence suggests a decline in overall HIV rates from 2010 to 2017, some populations continue to be at risk of HIV acquisition. Within the USA, most of the new HIV diagnoses in 2018 were among individuals aged 25-34 years, with the second highest level of new diagnoses being among individuals aged 13-24 years. ${ }^{1}$ Thus, youth and young adults
Strengths and limitations of this study

- The Youth to Telehealth and Text to Improve Engagement in Care (Y2TEC) intervention is the first known technology-based counselling intervention developed for youth and young adults living with HIV that integrates HIV treatment engagement, substance use and mental health in an effort to improve HIV care outcomes.

- The short individualised 20-30 minute sessions held via videoconferencing can increase intervention uptake and continuation.

- The intervention's use of teleconferencing may circumvent traditional barriers to accessing counselling.

- The intervention was developed and piloted in a large metropolitan area which may impact generalisability.

- Technical issues can occur during telehealth that could impact rapport and session acceptability, though they can be mitigated.

represent a population that is experiencing disparate rates of HIV infection. In addition to having higher rates of HIV infection, youth and young adults living with HIV (YLWH) are less likely to be engaged in HIV treatment or to reach viral suppression than other age groups. $^{2}$

YLWH also experience unique constellations of factors that are directly correlated to missed medication doses, including psychological distress and substance use. ${ }^{3}$ For example, YLWH experience more mental health challenges (eg, symptoms of anxiety and depression) than the general population. ${ }^{4}$ Despite this, YLWH may be hesitant to use mental health treatment services because of negative experiences with past mental health providers or difficulty accessing mental health services. ${ }^{5}$ Poor treatment adherence to 
antiretroviral therapy (ART) can also be associated with increased substance use or misuse. ${ }^{6}$ While mental health and substance use impact ART adherence, they occur in the context of other psychosocial stressors often experienced by YLWH, including lower socioeconomic status, unstable housing and experiences with stigma that could also negatively impact ART adherence. ${ }^{4}$

Despite these barriers, few counselling interventions exist that target mental health and substance use to improve ART adherence for YLWH. ${ }^{4}$ Interventions targeting these psychosocial factors for adults have demonstrated positive findings but may not be appropriate for YLWH as they do not account for the cultural norms and age-specific needs of this population. ${ }^{7}$ For example, previous research suggests that using technology to deliver mental health interventions for adults living with HIV is promising. ${ }^{8}$ However, there are no known interventions developed for YLWH that target ART adherence while addressing substance use and mental health issues. Additionally, given that youth are more likely to use technology than older adults, technology-based counselling methods for this population could be a promising new area of study. ${ }^{9}$

This paper outlines the Youth to Telehealth and Text to Improve Engagement in Care (Y2TEC) intervention, which fills this gap in research by combining technologybased counselling, an integrated behavioural health approach, and specific content tailored for YLWH. The aim of the current paper was to describe the development of the intervention and to outline the intervention in detail. Here we provide a brief description of the Y2TEC pilot study, followed by detailed information on the development of the intervention. Finally, the intervention is described in detail, adhering to the Template for Intervention Description and Replication (TIDieR) guidelines to explain the intervention with specificity and to allow for future replication. ${ }^{10}$

\section{METHODS}

\section{Design of pilot study}

The Y2TEC intervention provided video-based counselling services out of the University of California, San Francisco (UCSF). This intervention was developed and piloted through a randomised pilot trial (trial registration NCT03681145). Primary objectives of the trial were to examine the feasibility and acceptability of a video-counselling intervention with YLWH. Participants were 50 YLWH who were recruited via flyers, in person clinic outreach, online advertisement and participation in previous studies. Eligibility criteria included any youth and young adults (18-29 years of age) living in or receiving medical care in the San Francisco Bay Area who had access to a smartphone with text-messaging capabilities. The study was a single-site randomised pilot trial delivered to participants in two groups: intervention or waitlist control. Participants in each condition received video-counselling sessions during a 4-month active treatment phase, staggered by 4 months for those randomised to the waitlist control. Bidirectional text messages were also used for scheduling counselling sessions, appointment reminders, reinforcement of in-session goal setting, delivery of community resources including free community events, and answering participant questions during both the intervention and waitlist control phases of the study. A detailed study protocol has been published outlining the research plan for assessing feasibility and acceptability, as well as details on eligibility, consent and enrollment processes. ${ }^{7}$ Detailed key findings of the trial are published elsewhere and discussed in further detail in the discussion of the current paper. ${ }^{11}$

\section{Intervention development}

The Y2TEC intervention was developed through an iterative process including (1) formative research with YLWH and healthcare providers serving YLWH, (2) review of appropriate theoretical frameworks and approaches for behavioural change, (3) interdisciplinary collaboration and (4) patient and public involvement. The intervention was also periodically refined in response to participant and clinician feedback.

\section{1) Formative research with YLWH and healthcare providers serving YLWH}

Initially, formative research was conducted to understand factors influencing ART adherence and engagement in HIV care for YLWH and to determine how to leverage technology to address barriers to ART adherence and engagement in care. The team conducted a crosssectional survey with 101 YLWH, which revealed that mental health symptoms (including increased symptoms of depression, adverse childhood experiences, and past trauma) as well as substance use (marijuana and stimulants) among YLWH had a negative association with ART adherence. ${ }^{5}$ Additionally, qualitative interviews with 29 YLWH illuminated barriers that prevented YLWH from addressing mental health or substance use challenges (eg, a perceived lack of access to treatment). Based on these identified barriers, the Y2TEC intervention was developed to provide participants access to non-judgemental, readily available and responsive staff in a way that was convenient to access (eg, little to no travel time), and addressed concerns regarding confidentiality that could occur during traditional office visits.

To further understand how to address these barriers to ART adherence for YLWH, the study team additionally conducted 17 individual in-depth interviews with healthcare providers and clinic staff. ${ }^{12}$ Several ways that providers and clinic staff engaged YLWH in 'youth-friendly' healthcare to reduce barriers to ART adherence emerged: being flexible, offering services that address the unique needs of YLWH, increasing accessibility and providing services that were aligned with cultural norms for YLWH, such as the integration of technology into services.

In previous studies, YLWH have favoured the idea of using video-counselling as a method of engaging in ART adherence counselling. ${ }^{513}$ In a pilot study that provided 
African-American YLWH with a single sample videocounselling session, most participants reported liking the video-counselling format. Video-counselling sessions were reported to be more convenient and comfortable, and offered the ability to be more candid with providers about barriers to ART adherence. Participants also reported that a single session of adherence counselling improved their HIV knowledge and motivation to adhere to treatment, and provided them with skills to address barriers to non-adherence. ${ }^{13}$

\section{2) Theoretical framework and approaches for behavioural change}

In addition to formative research, the Y2TEC intervention was also informed by the information-motivationbehavioural (IMB) skills model. ${ }^{14}$ According to this model, information, motivation and behavioural skills are all necessary for individuals to make positive changes in their lives. HIV risk reduction can be achieved for individuals by (1) providing HIV prevention information, (2) increasing motivation to actively reduce HIV risk behaviours and (3) developing behavioural skills needed for HIV prevention. When information and motivationbuilding are provided together, they can encourage behavioural change.

To promote the change process outlined in the IMB model, the Y2TEC intervention integrated several approaches that served as techniques for improving information, increasing motivation and developing skills to promote behavioural change. Psychoeducation/ health education was used to provide information, ${ }^{15}$ Motivational interviewing (MI) was employed to increase motivation, ${ }^{16}$ and problem-solving therapy was used to support the development of behavioural skills. ${ }^{17}$ These combined approaches addressed the mental health, substance use and physical health needs of YLWH. These three clinical methodologies were selected for use in the Y2TEC intervention due to their close fit as mechanisms to address behavioural change through the IMB model. Psychoeducation and health education provide clients with foundational information on HIV and behavioural health, MI is used to elicit and enhance motivation for change, and problem-solving therapy helps clients learn new behavioural skills to navigate environmental challenges. Finally, to implement behavioural change, the behavioural skills (eg, remembering to take medication) are rehearsed and practised, then translated into real-life settings.

Psychoeducation is the process of educating the client about their mental health symptoms and available treatments. ${ }^{15}$ Providing education about mental and physical health fosters collaboration between counsellors and clients and can help clients become more informed, improve their attitudes, make educated healthcare decisions, and potentially improve their overall health. ${ }^{18}$ Psychoeducation has been included in numerous evidence-based interventions targeting a large range of mental health diagnoses including mood disorders and psychotic disorders. ${ }^{15}$
MI is a counselling approach for eliciting and enhancing clients' motivation for behavioural change. ${ }^{16}$ It has been proven effective with decreasing substance use and increasing other health-promoting behaviours. ${ }^{19} \mathrm{MI}$ is known to be a good tool for clients experiencing ambivalence or concerns about making positive changes. ${ }^{16}$ This approach uses a guiding style of communication, balancing reflective listening with education. MI relies on four components that are fundamental for maintaining the underlying 'spirit' of the approach: partnership, acceptance, compassion and evoking. First, a partnership must be developed and maintained between the counsellor and the client to create sessions that feel collaborative and position the client as the expert on themselves. Next, MI encourages counsellors to practise acceptance towards clients, demonstrated by empathy, seeing the world through the client's eyes and sharing in the client's experience. MI also requires counsellors to be compassionate by promoting the welfare of their clients. Finally, $\mathrm{MI}$ includes evocation, which is the assumption that individuals are equipped with the necessary skills to make changes in their lives, but it is the counsellor's job to help evoke these skills. ${ }^{16}$

Problem-solving therapy is a treatment that assists individuals in effectively dealing with current stressors. ${ }^{17}$ It is appropriate for addressing large stressors as well as the culmination of 'minor' stressors. Problem-solving therapy includes helping clients improve decision-making, identify possible solutions, and set and complete tangible behavioural goals.

\section{3) Interdisciplinary collaboration}

An interdisciplinary team consisting of a clinical pharmacist/researcher, a nursing researcher, clinical social worker and several clinical psychologists collaborated in intervention development. Members of the team had expertise in substance use and mental health treatment, knowledge on strategies for improving ART adherence, and experience working with YLWH and conducting video-counselling research. Team members worked collaboratively by participating in weekly (or more frequent) meetings during the initial stage of intervention development.

The interdisciplinary team discussed the overall intervention focus and structure, components, session length and dosage to identify the best conditions for the YLWH population and use of video-based counselling methods. The team established that brief, 20-30 minute sessions would be ideal, given the use of technological platforms and the age of the target population. The intervention was developed as a 12-session series to provide adequate dosage given the shorter sessions. After extensive consultation with the UCSF Telehealth Resource Centre and review of several technology platforms, a video-counselling platform (Zoom) and text-messaging software (Mosio) were agreed on by the team for their functionality, cost and data security. 
Additionally, the team aimed to design an intervention that could be tailored to each participant's acuity of substance use and mental health challenges. The intervention was structured to use the results of an initial survey (including measures of depression, post-traumatic stress disorder, drug and alcohol misuse, and HIV knowledge) to prescribe fewer or more sessions on these topics based on the participant's acuity. Additionally, the optional 'wildcard' sessions were added to create flexibility for participants experiencing challenges severe enough to require a crisis-focused session tailored to their needs rather than the scheduled session. For example, a wildcard session could include risk assessment and safety planning around suicidal ideation or other safety concerns. The decision to have wildcard sessions was based on clinical judgement and conversation with the participant at the beginning of session. Wildcard sessions were provided on an as-needed basis and took place at any time within the intervention, replacing one of the scheduled core or menu sessions, as needed.

\section{4) Patient and public involvement}

The intervention team also simultaneously consulted with a community advisory group composed of YLWH, called the Youth Advisory Panel (YAP), to gain critical insights into the priorities and preferences of YLWH with regard to mobile health technology for engagement in HIV care and ART adherence. The YAP expressed strong support for using a video-counselling platform and text messaging for the sessions. They further highlighted the interconnected relationship between engagement in HIV care, substance use, mental health challenges and other stressors (eg, family issues and housing instability). The YAP also advocated for an intervention with a holistic approach to their needs, including a focus on non-HIV-specific issues, such as romantic relationships, family disclosure and career aspirations. This information was used to develop the menu sessions of the intervention (described in detail as follows). The team continued to consult with the YAP for the duration of the pilot study through regular meetings.

The research team then created the intervention manual through an iterative process of team meetings, development of manual drafts by the team's social worker and re-review of the manual draft by all team members which lasted several months. Next, thorough training guidelines and resources were added, including areas related to assessment, safety planning and crisis response. A directory of community resources was added for each county where recruitment was planned.

\section{INTERVENTION COMPONENTS: GUIDED BY TIDIER GUIDELINES}

The intervention components are described as follows, guided by the TIDieR gidelines (seeonline supplemental figure 1).

\section{Rationale}

Due to the association between HIV care engagement, mental health and substance use, the Y2TEC intervention takes an integrative approach at addressing these three areas. The intervention aims to help participants increase their understanding of the interplay between these three concerns and address related barriers that may be impacting their health and well-being. The target outcomes of the intervention are increased engagement in HIV care and HIV viral suppression. To achieve these outcomes, behaviours addressed in sessions include taking medications, increasing attendance to healthcare appointments and completing laboratory testing.

\section{Intervention materials and procedures}

Materials and mode of delivery

The Y2TEC intervention consists of 12 sessions, each lasting 20-30 minutes in length. Counsellors deliver each session while closely following the intervention manual, which provides the topics to be covered in each session, sample wording for commonly discussed topics, guidance for topics such as confidentiality and safety/risk assessment, and training objectives for future counsellors. The intervention sessions are completed via teleconferencing, using a secure video-counselling platform (eg, Zoom) that can be accessed by participants via a computer, tablet or smartphone. Participants additionally receive text messages through a Health Insurance Portability and Accountability Act (HIPAA) compliant platform. Participants receive a text message appointment reminder the day before their appointment and an additional text message reminder with the video-counselling meeting link 15 min before the session. Additional text messages with community resources (general and any specific resources requested) are sent between sessions. ${ }^{7}$

\section{Procedures: assessments and initial session}

The intervention begins with participants completing an online baseline survey including questions about their mental health, substance use and HIV care. Mental health measures include the Patient Health Questionnaire-9 (PHQ-9) ${ }^{20}$ the Post-traumatic Stress Disorder Checklist (PCL-5), ${ }^{21}$ and a seven-item Generalised Anxiety Disorder Scale-7 (GAD-7). ${ }^{22}$ Substance use measures are the Alcohol Use Disorders Identification Test (AUDIT), ${ }^{23}$ the Alcohol, Smoking and Substance Involvement Screening Test (ASSIST) ${ }^{24}$ and the Drug Abuse Screening Test (DAST) ${ }^{25}$ For HIV knowledge, the HIV Treatment Knowledge Scale is used. ${ }^{26}$ Composite acuity scores are automatically calculated by the survey platform, which are then emailed to the counsellor.

Participants then complete an initial video-counselling session where the counsellor or research assistant builds rapport and orients the participant to the intervention. The research assistant helps the participant download the videoconferencing application, provides an overview of the application, and troubleshoots any initial technical or privacy concerns. To protect the participant's privacy, the counsellor informs the participants that they should be in a private location (which the counsellor inquires about at the beginning of each session) and to use headphones 
Table 1 Topics of initial session

\begin{tabular}{ll}
\hline Areas to explore during initial session \\
\hline Areas to explore & Example topics \\
\hline Physical/medical history & $\begin{array}{l}\text { Significant health conditions } \\
\text { impacting daily life }\end{array}$ \\
HIV and HIV treatment history & $\begin{array}{l}\text { HIV medications started, } \\
\text { stopped and missed }\end{array}$ \\
\hline Psychiatric history & $\begin{array}{l}\text { Mental health diagnoses and } \\
\text { current symptom severity }\end{array}$ \\
\hline Substance use history & $\begin{array}{l}\text { Current and past substance } \\
\text { use }\end{array}$ \\
\hline Housing situation & $\begin{array}{l}\text { History of unstable housing } \\
\text { or homelessness }\end{array}$ \\
Work, school, financial & $\begin{array}{l}\text { Occupational or student } \\
\text { status or goals }\end{array}$ \\
situation & $\begin{array}{l}\text { Friends and social support, } \\
\text { romantic and sexual } \\
\text { relationships }\end{array}$ \\
\hline Family relationships & $\begin{array}{l}\text { Family or origin or chosen } \\
\text { family }\end{array}$ \\
\hline $\begin{array}{l}\text { Stigma/discrimination } \\
\text { Strperiences }\end{array}$ & $\begin{array}{l}\text { Stigma/discrimination } \\
\text { oxperiences due to sexual } \\
\text { orientation, gender identity } \\
\text { and disability }\end{array}$ \\
\hline
\end{tabular}

when necessary during sessions. The counsellor then completes an initial assessment (table 1) that was adapted from a behavioural intervention for people living with HIV, to tailor the intervention to the participant. ${ }^{27}$ Assessment topics include the participant's HIV care, mental health and substance use history, as well as any other factors that could be impacting their HIV care and ART adherence.

\section{Procedures: intervention tailoring and high-acuity and low-acuity core sessions}

In tandem with the narrative assessment, the results of the surveys completed at enrollment are used to assess participant's needs and individualise the intervention for each participant (figure 1). From the initial survey, high HIV care acuity was defined by the study team as low HIV knowledge (indicated by a score of 12 or less on the HIV Treatment Knowledge Scale), a detectable viral load, lack of ART adherence, or no appointments with a healthcare provider in the past 6 months and no upcoming appointments scheduled.

Participants with a high acuity score receive two core sessions related to HIV engagement, with the first session including a more detailed assessment of barriers (see online supplemental material, Example of sessions, pages 1-3). Guided by the 'motivation' component of the IMB model, the goal of this first session is to help participants gain essential knowledge, self-awareness and motivation that is a prerequisite for any behavioural change. This session begins similarly to other sessions in the intervention, with the counsellor providing a greeting, ensuring the participant is able to access the videocounselling platform and confirming the participant's location, ensuring that it is sufficiently private. Next, the counsellor conducts an assessment of barriers to HIV treatment engagement. This is completed through a series of questions related to topics such as current acceptance/understanding of diagnosis, stigma-related beliefs related to HIV, past experiences/current thoughts about healthcare, current medication regimens and strengths/ challenges related to HIV care. The session then focuses specifically on enhancing motivation to address those barriers. The session also addresses the 'information' component of the IMB model by providing education on HIV treatment (via review of the HIV Treatment Knowledge Scale) and engaging the participant in discussion about how to address barriers to care. The second core HIV session (which all participants receive; see online supplemental material, Example of sessions, pages 4-6) focuses more broadly on HIV and health education (ie, information section of the IMB model). Counsellors assess for potential barriers to HIV treatment engagement and review basic information about HIV and HIV care (eg, attending clinic visits, brief overview of HIV pharmacology, ART adherence, completion of labs and medical literacy) that is targeted to address the identified barriers. The session ends with enhancing the patient's motivation for behavioural change by discussing ways they can address the identified barriers.

This method of individualising the intervention is also used for the mental health and substance use core sessions. Individuals who have an elevated score (indicating high acuity) on the PHQ-9 (more than 10), an elevated score on the PCL-5 (more than 33) or an elevated score on the GAD-7 (more than 10) receive two mental health core sessions (with the first one focusing primarily on enhancing motivation to address mental health-related barriers), while individuals who do not have elevated scores receive only one (focused on providing psychoeducation and motivation enhancement). Similarly, individuals who report elevated scores (e.g., high acuity) on the AUDIT (more than 8), or who indicate monthly use of drugs other than marijuana on the ASSIST, daily use of marijuana or cigarettes on the ASSIST, or who reported an elevated score (three or more) on the DAST receive two substance use core sessions. Individuals without elevated scores on these measures receive one. The two core sessions for mental health and substance use follow a similar pattern to the HIV core sessions. The first session (provided to participants with high acuity only) is designed to address the 'motivational' component of the IMB model. It begins with the counsellor assessing for any challenges/barriers related to mental health or substance use through a review of the elevated survey measures and discussion with the participant. The session then focuses on building motivation to address substance use and/or mental health-related barriers that, if resolved, would result in a positive impact on health and overall life satisfaction. The second mental health and substance use core sessions 


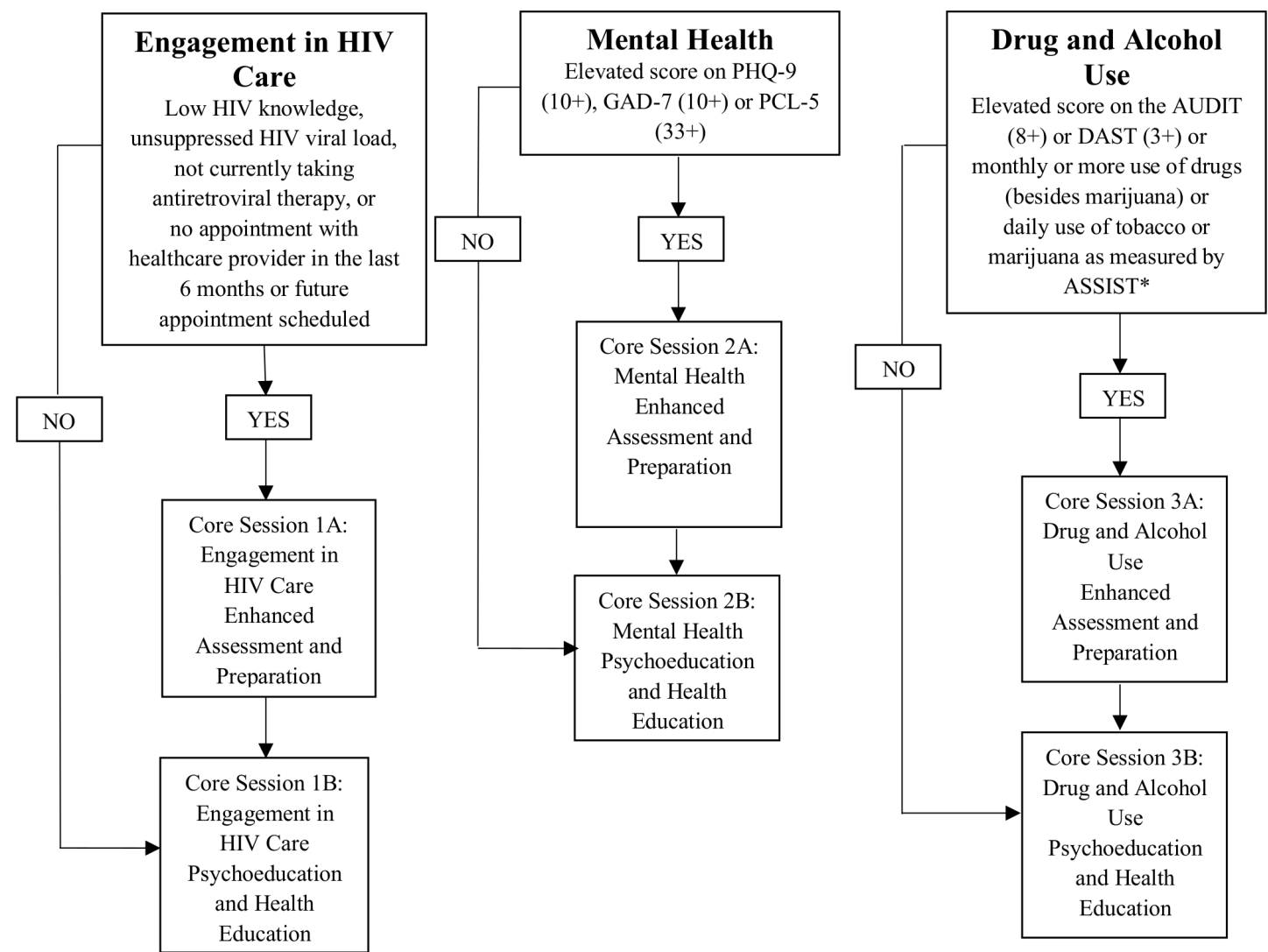

Figure 1 Outline of core session individualisation and content based on self-report measures. *Note: only item 2 on the ASSIST was used in order to assess for substance use patterns over the past 3 months. ASSIST, Alcohol, Smoking and Substance Involvement Screening Test; AUDIT, Alcohol Use Disorders Identification Test; DAST, Drug Abuse Screening Test; GAD-7, Generalised Anxiety Disorder Scale-7; PCL-5, Post-traumatic Stress Disorder Checklist - 5; PHQ-9, Patient Health Questionnaire-9.

(provided to every participant) address the information portion of the IMB model and start with an assessment of any health-related barriers related to mental health or substance use. The counsellor then provides education related to the barrier (e.g., how to identify mental health symptoms and treatment options and resources for managing risk related to drug/alcohol use).

This results in each participant receiving a minimum of three core sessions in HIV care, mental health and substance use but up to six if they experience higher acuity in these areas. If participants reported little or no issues in HIV care engagement, mental health and/ or substance use, they are provided with the minimum of three core sessions (one on each topic) which focus on reinforcing the participant's behaviour and success in maintaining this behaviour in the future.

\section{Procedure: remaining sessions (menu sessions)}

Following the first initial rapport-building session and three to six core sessions, participants receive four to seven more 'menu' sessions that focus on the behavioural component of the IMB model (table 2). Topics of these sessions are based on formative research and current population needs in the San Francisco Bay Area. Each of these session topics can be repeated as needed. After starting the session and ensuring privacy, the counsellor elicits information about the participant's chosen focus area or 'menu option' (eg, lack of social support; see online supplemental material, Example of sessions, pages $7-8)$. The participant is then guided to identify a barrier related to the focus area that may be impacting their HIV treatment and ART adherence or overall health (eg, a lack of social support from other people with HIV leading to shame). After a barrier has been identified, feedback and education are provided by the counsellor (eg, informing the participant of support groups).

The session ends with the participant setting a goal of how to address the identified barrier and building motivation to reach the goal. The goal should follow the SMART (specific, measurable, attainable, relevant and time-bound) goal format. ${ }^{28}$ For example, a participant may agree to attend one HIV support group over the next week. Using a readiness ruler from 0 to 10 (stemming from MI), the participant rates the importance of and self-confidence in reaching their goal. Following the session, the counsellor may text message the participant additional resources as needed, such as a list of local support groups. Later in the week, the counsellor checks on progress towards the identified goal through an automated text message prompt. The counsellor then discusses the participant's goal at the beginning of the subsequent session. 
Table 2 Topics for menu sessions

\begin{tabular}{|c|c|}
\hline Topics & Goals \\
\hline HIV care (in depth) & $\begin{array}{l}\text { Health literacy, communication and problem-solving skills needed to effectively and routinely } \\
\text { access HIV care }\end{array}$ \\
\hline Substance use (in depth) & $\begin{array}{l}\text { Accessing resources, enhancing motivation and increasing problem-solving skills needed to help } \\
\text { reduce or manage drug or alcohol use }\end{array}$ \\
\hline Social support & $\begin{array}{l}\text { Effective communication and problem-solving skills needed to maintain long-term supportive } \\
\text { social relationships }\end{array}$ \\
\hline Family of origin & $\begin{array}{l}\text { Effective communication and problem-solving skills needed to help have healthy relationships with } \\
\text { their families of origin }\end{array}$ \\
\hline Subsistence needs & Addressing access to material and financial resources and stability \\
\hline Education and vocation & Planning for current or future educational/vocational goals \\
\hline Wildcard & Problem-solving support to help address serious barriers and safety concerns \\
\hline
\end{tabular}

After the predetermined core and menu topic sessions are completed, the counsellor and the participants engage in a final 12 th session. To reinforce motivation and build self-efficacy, participants review the most impactful topics from their work with the counsellor, discussing their life changes, successes and goal completion in each topic. The counsellor and participant then discuss the participant's continuing goals and make a change maintenance plan. The final session ends with the counsellor thanking the participants for their participation. While the counsellor can provide the participants with additional community resources (eg, long-term mental health or substance use resources) on an as-needed basis at any time during the intervention, the counsellor focuses on providing any additional resources the participants may need in the final session to facilitate appropriate treatment linkage.

\section{Role of counsellor and training requirements}

Sessions are delivered by a trained mental health professional (eg, clinical social worker, clinical psychologist or another psychotherapist) who is referred to as the 'counsellor'. Counsellor training, spanning approximately 25 hours, is conducted to ensure fidelity of intervention delivery. The trainee begins by reviewing published formative research associated with the study and the Y2TEC intervention manual. ${ }^{57121329}$ The trainee is then asked to practice using the teleconferencing and text-messaging platforms, troubleshooting any issues in real time.

The trainee then reviews each session alongside an experienced counsellor, highlighting important areas (eg, session overview and content), discussing ways to tailor the session for each participant depending on level of education and baseline understanding of a topic, and providing other information about how to conduct each session. Next, each session is role-played with the trainee acting as a participant and an experienced counsellor serving as a counsellor. Prepared vignettes are used to represent experiences similar to those reported by real participants in the intervention. For example, a vignette might focus on a 20-year-old queer man who recently moved away from his hometown and is trying to become independent and learn how to manage his own healthcare in a new city.

After the trainee has observed each session, the trainee then assumes the role of a counsellor working with a participant, played by an experienced counsellor. Finally, the trainee is asked to practice each session independently with another project staff member (eg, research assistant) acting as the participant. All sessions are video-recorded, and the experienced counsellor and other study staff members (eg, clinical team members) review the recordings prior to the trainee providing the intervention with real participants. New counsellors begin seeing up to five participants per week, gradually increasing their caseload to full capacity (approximately 30 participants for a fulltime counsellor). Throughout the course of the intervention, all counsellors engage in weekly supervision and receive support from other experienced counsellors and the research team.

\section{Modifications}

Manual modifications during the intervention were based on participant experiences and feedback to further tailor the intervention for YLWH. For example, community resources were included in the manual, which could be provided to participants at the end of sessions when 
appropriate. Additionally, sessions were modified to be more appropriate for participants with unique needs. For example, to be more appropriate for pregnant participants, the HIV treatment engagement sessions were modified to include information on HIV and breast feeding, when necessary.

\section{DISCUSSION}

The pilot test of the Y2TEC intervention was completed in November 2019. The acceptability of the intervention was measured through participant satisfaction questions at the end of each session and at the end of the intervention with a detailed 30-item questionnaire. The intervention was examined for feasibility, assessed through recruitment and retention rates as well as by the number of disconnections that occur during each session, participants' text-message response time and the counsellor's post-session ratings of the session's sound and video quality. Results indicated a high level of feasibility and acceptability of the intervention. Preliminary evidence on effectiveness also indicated improvement in ART adherence, HIV knowledge, mental health symptoms, and stigma related to mental health and substance use problems 4 months following the intervention. ${ }^{11}$ Fidelity to the intervention was assessed during weekly meetings with the counsellors to review each session's length, technical issues, topics covered, goals established and narrative progress notes. Counsellors also completed a fidelity checklist for each session to determine if the focus area was identified, if education/information was provided, if barriers were identified, if motivation was enhanced and if problem solving was initiated. These fidelity checklists were reviewed regularly by a study coinvestigator.

The Y2TEC intervention holds potential for addressing the unique mental health, substance use and HIV treatment engagement needs of the young adult population that is disproportionately impacted by the HIV epidemic. ${ }^{12}$ The intervention uses technology to provide counselling that is congruent with the cultural norms and technology preferences of YLWH to promote the health and well-being of this underserved community. Taking a novel approach, the Y2TEC intervention does not solely focus on HIV treatment engagement but also additionally focuses on mental health and substance use, as these are factors that have been shown to impact HIV care for YLWH. Recognising that these issues occur in the context of other psychosocial stressors, the Y2TEC intervention takes a holistic approach, offering participants guidance on topics such as social support, stigma and disclosure to improve engagement in HIV treatment.

While the Y2TEC counselling series is promising, there are some limitations that could impact large-scale implementation of the intervention. First, the intervention was developed and piloted in a large metropolitan area of San Francisco Bay Area; it has not yet been tested for feasibility and acceptability in other communities. Another limitation is that technological issues occurred during intervention sessions that could impact rapport and session acceptability. However, these technological issues were identified and addressed within session to reduce negative impact, as described in an article by this team that focuses on overcoming technological issues in videobased counselling. ${ }^{29}$

Despite potential limitations, the Y2TEC intervention demonstrates several strengths. The intervention's use of teleconferencing may circumvent traditional barriers to accessing counselling (e.g., living in an isolated location, no access to transportation and stigma related to attending in-person sessions), which could make it more accessible to populations most at risk of not being engaged in HIV treatment services. Additionally, the individualised nature of the intervention can provide a patient-centred experience that might improve clinical outcomes. The short 20-30 minute sessions held via videoconferencing can increase intervention uptake and continuation in several different treatment settings, such as medical offices or social services agencies, as clinician burden is low. Finally, the Y2TEC intervention is the first known technology-based counselling intervention developed for YLWH that integrates not only HIV treatment engagement but also substance use and mental health in an effort to improve HIV care outcomes.

In conclusion, the Y2TEC intervention is a promising 12-session videoconferencing counselling intervention aimed at improving HIV treatment engagement and ART adherence for YLWH. The study's use of video-counselling technologies is a novel application to this population and may prove effective and acceptable to youth. Findings from this intervention will inform the development of new interventions and dissemination of similar interventions to help decrease HIV-related disparities in YLWH.

\section{Author affiliations}

${ }^{1}$ Department of Psychiatry and Behavioral Sciences, University of California, San Francisco, San Francisco, California, USA

${ }^{2}$ School of Social Welfare, University of California, Berkeley, Berkeley, CA, USA ${ }^{3}$ Center for AIDS Prevention Studies, Department of Medicine, University of California, San Francisco, San Francisco, California, USA

${ }^{4}$ Department of Community Health Systems, School of Nursing, University of California, San Francisco, San Francisco, California, USA

Acknowledgements Publication made possible in part by support from the UCSF Open Access Publishing Fund.

Contributors CM took the primary role of manuscript preparation. AW and VG are familiar with the clinical aspects of the intervention and reviewed the intervention description thoroughly. DLP, MJ and CDR provided guidance on manuscript preparation and scientific writing. PS has oversight of intervention development and the pilot study and served as corresponding author. All authors read and approved the final manuscript.

Funding The study is funded by the California HIV/AIDS Research Program (award number HD15-SF-060) (coprincipal investigators PS and CDR) and the National Institutes of Health (award number K24DA037034, MJ).

Competing interests None declared.

Patient consent for publication Not required.

Ethics approval All study procedures for the trial were approved by the UCSF Institutional Review Board.

Provenance and peer review Not commissioned; externally peer reviewed. 
Data availability statement The datasets used and/or analyzed during the current study are available from the corresponding author on reasonable request

Supplemental material This content has been supplied by the author(s). It has not been vetted by BMJ Publishing Group Limited (BMJ) and may not have been peer-reviewed. Any opinions or recommendations discussed are solely those of the author(s) and are not endorsed by BMJ. BMJ disclaims all liability and responsibility arising from any reliance placed on the content. Where the content includes any translated material, BMJ does not warrant the accuracy and reliability of the translations (including but not limited to local regulations, clinical guidelines, terminology, drug names and drug dosages), and is not responsible for any error and/or omissions arising from translation and adaptation or otherwise.

Open access This is an open access article distributed in accordance with the Creative Commons Attribution Non Commercial (CC BY-NC 4.0) license, which permits others to distribute, remix, adapt, build upon this work non-commercially, and license their derivative works on different terms, provided the original work is properly cited, appropriate credit is given, any changes made indicated, and the use is non-commercial. See: http://creativecommons.org/licenses/by-nc/4.0/.

\section{ORCID iDs}

Caravella McCuistian http://orcid.org/0000-0002-1898-2431

Angie R Wootton http://orcid.org/0000-0003-1689-4793

Parya Saberi http://orcid.org/0000-0002-3793-5112

\section{REFERENCES}

1 CDC. Hiv in the United States and dependent areas, 2020. Available: https://www.cdc.gov/hiv/statistics/overview/ataglance.html [Accessed 3 Jan 2020].

2 CDC. Hiv and youth, 2019. Available: https://www.cdc.gov/hiv/ group/age/youth/index.html [Accessed 3 Jan 2020].

3 MacDonell K, Naar-King S, Huszti H, et al. Barriers to medication adherence in behaviorally and perinatally infected youth living with HIV. AIDS Behav 2013;17:86-93.

4 Vreeman RC, McCoy BM, Lee S. Mental health challenges among adolescents living with HIV. J Int AIDS Soc 2017;20:21497-9.

5 Saberi P, Dawson Rose C, Wootton AR, et al. Use of technology for delivery of mental health and substance use services to youth living with HIV: a mixed-methods perspective. AIDS Care 2020;32:931-9.

6 Gamarel KE, Brown L, Kahler CW, et al. Prevalence and correlates of substance use among youth living with HIV in clinical settings. Drug Alcohol Depend 2016;169:11-18.

7 Wootton AR, Legnitto DA, Gruber VA, et al. Telehealth and Texting intervention to improve HIV care engagement, mental health and substance use outcomes in youth living with HIV: a pilot feasibility and acceptability study protocol. BMJ Open 2019;9:e028522-11.

8 Kempf M-C, Huang C-H, Savage R, et al. Technology-Delivered mental health interventions for people living with HIV/AIDS (PLWHA): a review of recent advances. Curr HIVIAIDS Rep 2015;12:472-80.

9 Olson KE, O'Brien MA, Rogers WA, et al. Diffusion of technology: frequency of use for younger and older adults. Ageing Int 2011;36:123-45.
10 Hoffmann TC, Glasziou PP, Boutron I, et al. Better reporting of interventions: template for intervention description and replication (TIDieR) checklist and guide. BMJ 2014;348:g1687-12.

11 Saberi P, McCuistian C, Agnew E, et al. Video-Counseling intervention to address HIV care engagement, mental health, and substance use challenges: a pilot randomized clinical trial for youth and young adults living with HIV. Telemed Rep 2021;2:14-25.

12 Saberi P, Ming K, Dawson-Rose C. What does it mean to be youth-friendly? Results from qualitative interviews with health care providers and clinic staff serving youth and young adults living with HIV. Adolesc Health Med Ther 2018;9:65-75.

13 Saberi $P$, Yuan $P$, John $M$, et al. A pilot study to engage and counsel HIV-positive African American youth via telehealth technology. AIDS Patient Care STDS 2013;27:529-32.

14 Fisher JD, Fisher WA. Changing AIDS-Risk behavior, 1992: 455-74.

15 Lukens EP, McFarlane WR. Psychoeducation as evidence-based practice: considerations for practice, research, and policy. Brief Treat Crisis Interv 2004;4:205-25.

16 Miller WR, Rollnick S. The spirit of motivational interviewing. In: Motiv Interviewing Prep People Chang, 2013: 14-24. https://ereserves. library.queensu.ca/ares/ares. dll? Action=10\&Type $=10 \&$ Value $=98979 \%$ OAhttps://ereserves.library.queensu.ca/ares/ares.dll?Action=10\& Type $=10 \&$ Value $=58283$

17 Nezu AM, Nezu CM, D'Zurilla T. Problem-Solving therapy: a treatment manual. New York, NY, 2013.

18 World Health Organization. Health education, 2020. Available: https:// www.who.int/topics/health_education/en/

19 Lundahl BW, Kunz C, Brownell C, et al. A meta-analysis of motivational interviewing: twenty-five years of empirical studies. Res Soc Work Pract 2010;20:137-60.

20 Spitzer RL, Kroenke K, Williams JBW. Validation and utility of a selfreport version of PRIME-MD. Prim Care Companion J Clin Psychiatry 2000;2:31.

21 Weathers FW, Litz BT, Keane TM. The PTSD checklist for DSM-5 (PCL-5), 2013.

22 Spitzer RL, Kroenke K, Williams JBW, et al. A brief measure for assessing generalized anxiety disorder: the GAD-7. Arch Intern Med 2006;166:1092-7.

23 Bohn MJ, Babor TF, Kranzler HR. The alcohol use disorders identification test (audit): validation of a screening instrument for use in medical settings. J Stud Alcohol 1995;56:423-32.

24 Humeniuk R, Ali R, Babor TF, et al. Validation of the alcohol, smoking and substance involvement screening test (ASSIST). Addiction 2008;103:1039-47.

25 Skinner HA. The drug abuse screening test. Addict Behav 1982;7:363-71.

26 Balfour L, Kowal J, Tasca GA, et al. Development and psychometric validation of the HIV treatment knowledge scale. AIDS Care 2007;19:1141-8.

27 The Healthy Living Project Team. Effects of a behavioral intervention to reduce risk of transmission among people living with HIV. JAIDS $J$ Acquir Immune Defic Syndr 2007;44:213-21.

28 Doran GT. There's a S.M.A.R.T. Way to Write Management's Goals and Objectives. Manage Rev 1981;70:35-6.

29 Wootton AR, McCuistian C, Legnitto Packard DA, et al. Overcoming technological challenges: lessons learned from a telehealth counseling study. Telemed J E Health 2020;26:1278-83. 